\title{
Le théâtre antique de Lousonna-Vidy
}

\section{François Eschbach}

\section{(2) OpenEdition}

Journals

Édition électronique

URL : http://journals.openedition.org/edl/106

DOI : $10.4000 /$ edl. 106

ISSN : 2296-5084

\section{Éditeur}

Université de Lausanne

\section{Édition imprimée}

Date de publication : 15 mai 2011

Pagination : 97-118

ISBN : 978-2-940331-25-3

ISSN : 0014-2026

\section{Référence électronique}

François Eschbach, "Le théâtre antique de Lousonna-Vidy », Études de lettres [En ligne], 1-2 | 2011, mis en ligne le 15 mai 2014, consulté le 19 décembre 2020. URL : http://journals.openedition.org/edl/106 DOI : https://doi.org/10.4000/edl.106 


\section{LE THÉÂTRE ANTIQUE DE LOVSONNA-VIDY}

La découverte inattendue du théâtre gallo-romain de Lousonna-Vidy a mis en évidence des particularités notables, soulignant tout son intérêt. Elles permettent de soulever nombre de problématiques nouvelles comme l'influence d'une forme de monument importée sur la tradition locale du spectacle. C'est également l'occasion d'évoquer des thèmes non résolus de façon satisfaisante: la typologie des théâtres, les liens entre la forme du monument et les spectacles qui peuvent y être présentés. Abordés ici superficiellement, ils stimulent la poursuite de l'étude sur les monuments de spectacles.

\section{Introduction}

\subsection{Circonstances de la découverte et déroulement des travaux}

Le théâtre gallo-romain de Lousonna a été découvert à la faveur d'un projet immobilier, au bas de l'avenue des Figuiers. En 1894, lors de fouilles ponctuelles, quelques murs, sans doute rattachables à l'enceinte du théâtre, avaient déjà été mis au jour. Les opérations, réalisées un siècle plus tard avec d'énormes engins, ont permis d'atteindre des vestiges insoupçonnés, environ $3 \mathrm{~m}$ sous les remblais. Cette découverte doit beaucoup à la vigilance des archéologues, sans laquelle l'affaire du théâtre eût été réglée en une demi-journée.

Le travail a principalement consisté à dégager les vestiges subsistants de leur gangue limoneuse. Rapidement, décision fut prise de conserver le monument sous le futur bâtiment. De ce choix - favorable à la conservation des ruines - et de l'exiguiité des surfaces fouillées sont nées 
des lacunes dans le dossier scientifique où les hypothèses, aujourd'hui encore, restent plus nombreuses que les certitudes.

\subsection{Aperçu géographique et stratigraphique général}

La configuration topographique actuelle ne correspond guère à la géomorphologie locale. Le talus abrupt sous lequel ont été trouvés les vestiges cache en réalité une côte de faible pendage, s'étirant jusque sur les rives du lac Léman, à $300 \mathrm{~m}$ de là (fig. 1). Les coupes réalisées parallèlement à la pente montrent des couches stratigraphiques n'excédant généralement pas un pendage de $8,5^{\circ}$, comparable à celui de l'ensemble des gradins conservés. Dans le sens est-ouest, un faible relèvement des couches dans des directions opposées indique la présence d'une légère cuvette topographique.

L'ensemble des couches appartient à des dépôts morainiques et marginalement à l'ancien delta du Flon. Ces matériaux sont très fluants même sur une faible pente. Ceci explique la disparition de la plupart des niveaux de circulation antiques. L'influence des mouvements de terrain est également ressentie sur les vestiges eux-mêmes: les murs sont fortement déversés ou partiellement effondrés. Les terrassements antiques compliquent encore la vision globale: les matériaux enlevés à la colline et replacés vers l'avant ou sur les côtés du monument sont identiques à ceux encore en place. Quoi qu'il en soit, au début du II siècle apr. J.-C., la configuration est suffisamment favorable pour établir un théâtre et profiter de la pente pour y aménager des gradins, offrant un panorama dégagé sur le lac Léman.

\subsection{Localisation du théâtre}

Le théâtre a été édifié sur la rive gauche du Flon, à plus de $400 \mathrm{~m} \mathrm{du}$ forum de Lousonna (fig. 1), soit en dehors du périmètre urbain proprement dit ${ }^{1}$. Une localisation périphérique peut avoir plusieurs motifs allant de l'éloignement des nuisances aux contraintes urbanistiques.

Si la tendance générale est d'implanter le théâtre dans les quartiers centraux, on constate, en Gaule principalement, de nombreuses

I. Cette remarque est toutefois à pondérer après la découverte d'un quartier d'habitat antérieur au théâtre: cf. F. Eschbach, C. Brunetti, Vidy, av. des Figuiers 41 et 43. 


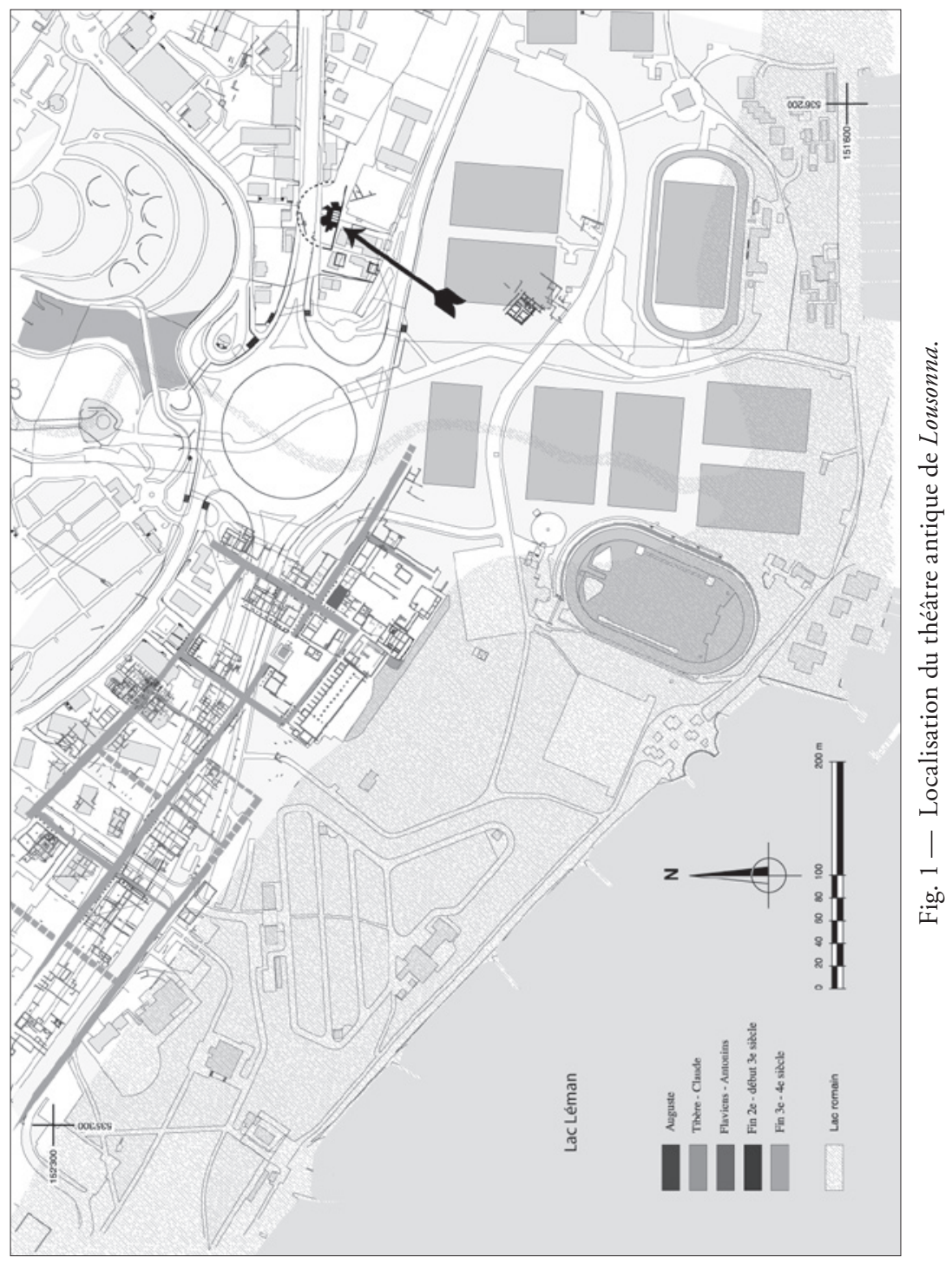


exceptions dictées soit par un espace insuffisant, soit par des conditions topographiques plus favorables en périphérie, soit encore par un choix délibéré ${ }^{2}$. A Lousonna, les constructeurs, tenus à l'écart du centre par un développement urbain déjà grandement réalisé, ont mis à profit la pente naturelle du site pour établir leur édifice avec un minimum de travaux de terrassement, en économisant une partie des substructures ${ }^{3}$. Les matériaux excavés dans la pente de la colline sont réutilisés à l'avant pour l'élévation latérale des rangées de gradins. Plus prosaïquement, on conçoit que la construction d'un théâtre ne fait pas partie des premières réalisations urbanistiques du vicus, dont l'activité est orientée vers le commerce et le transport des marchandises.

Des justifications religieuses peuvent être évoquées. Certains théâtres «ruraux» de Gaule sont associés à des bâtiments religieux (temples) et ce en dehors du périmètre construit (Argenton, Champlieu, Genainville, Lenzburg, etc.). Ce n'est pas le cas à Vidy où aucun temple n'a été reconnu.

Bien qu'isolé du centre actif, le théâtre de Lousonna est certainement situé à proximité des principaux axes de circulation. Toutefois, aucun élément du réseau de voirie n'a été repéré sur le site et l'on peut exclure le passage d'une voie directement au sud du théâtre. Le lien entre les quartiers est et ouest de l'agglomération demeure inconnu. Celui-ci était certainement établi par un pont, jeté au-dessus du Flon, qu'empruntait le trafic routier en direction des cols du Valais, vers l'Italie.

L'orientation du théâtre de Vidy, pratiquement plein sud, est également une particularité ${ }^{4}$, fortement déconseillée par Vitruve ${ }^{5}$. Ce dernier fait allusion au risque de surchauffe de la cavea, auquel s'ajoutent les désagréments visuels liés à la position du soleil, les spectacles se jouant en plein jour, dès le matin.

2. Choix qui peut reposer sur des motivations politiques ou religieuses. A Avenches par exemple, on note un éloignement du théâtre de $400 \mathrm{~m}$ au centre, mais il s'agit d'un ensemble cultuel qui exige une surface considérable.

3. A Autun, en construisant le théâtre à plus de $700 \mathrm{~m}$ du centre, les architectes ont avant tout choisi de profiter des dénivellations à l'est de l'agglomération. A Argenton, le théâtre est bâti à $400 \mathrm{~m}$ du centre, principalement pour des commodités de construction; cf. F. Dumasy, Le théatre d'Argentomagus (Saint-Marcel, Indre), p. 44. C'est le cas également (partiellement du moins) à Augst, Lyon, Lyons-la-Forêt, Vienne, etc.

4. D'autant plus surprenante en l'absence de mur de scène.

5. Vitruve, De Architectura 3.2. 
Il est intéressant de souligner que le théâtre respecte une trame orthogonale préexistante qui régit l'implantation d'un quartier d'habitation antérieur à sa construction ${ }^{6}$.

\section{Le théâtre}

Le théâtre de Lousonna est le quatrième monument de ce genre découvert en Suisse. Avec $60 \mathrm{~m}$ de diamètre, il ferme la marche, loin derrière celui d'Avenches et ses $110 \mathrm{~m}$. Il présente des analogies avec celui de Lenzburg $^{7}$, particulièrement au niveau de la conception des gradins. Les édifices d'Augst et d'Avenches, tous deux productions de grandes cités possédant le statut de colonie romaine, sont des constructions dont le caractère monumental est nettement plus appuyé. Cela se traduit notamment par un traitement architectural plus abouti, mettant en œuvre des techniques complexes pour un résultat délibérément imposant.

Le monument a été dégagé sur environ un tiers de sa superficie. Les vestiges mis au jour montrent un état général de conservation médiocre. Les glissements de terrain, la récupération d'une grande partie des éléments de construction et enfin des travaux publics modernes fortement perturbants empêchent une restitution claire du bâtiment.

Les ruines se résument à un long mur frontal rectiligne, orienté sur un axe est-ouest prolongé d'un retour vers le nord à son extrémité ouest, à quelques rangs de gradins de molasse disposés (ou non) sur des structures rayonnantes d'orientations divergentes et aux substructures de la scène. Le tout est très arasé.

\subsection{Problèmes de typologie}

Les particularités du théâtre de Lousonna le rendent difficile à classer dans une typologie aujourd'hui à redéfinir ${ }^{8}$. Jusqu'ici, on reconnaissait

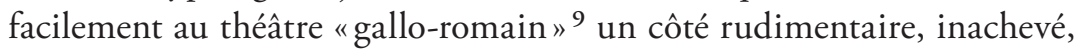

6. Fouille de la parcelle contiguë, cf. F. Eschbach, C. Brunetti, Vidy, av. des Figuiers 41 et 43.

7. U. Niffeler, Römisches Lenzburg.

8. En effet, la typologie actuelle ne fait pas l'unanimité chez les auteurs.

9. Cette appellation est elle-même remise en question pour son manque de précision. 
par opposition au "théâtre classique" de conception architecturale beaucoup plus aboutie et lourdement chargée politiquement.

La spécificité du théâtre "gallo-romain" se mesure à la modification de certaines composantes ${ }^{10}$ par rapport au modèle idéal du théâtre romain "classique» défini par Vitruve. Toutefois, le manque d'édifices bien étudiés ${ }^{11}$ et l'état de conservation des vestiges handicapent fortement l'établissement de critères suffisamment précis pour réaliser des comparaisons significatives. Les documents anciens proposent des représentations schématiques, voire idéalisées. La fouille des monuments importants a longtemps été axée sur une restitution du plan et une mise en valeur des vestiges, parfois au détriment de la recherche scientifique.

Le concept est d'autant plus difficile à saisir que tous les théâtres recensés sous l'appellation "gallo-romain» ne sont pas situés en Gaule ${ }^{12}$ et que l'on trouve des théâtres "classiques" en Gaule comme ailleurs. La confusion qui règne sur les critères définissant chaque type et les querelles d'auteurs compliquent encore le problème. En outre, les découvertes récentes soulignent certaines caractéristiques. La pente des gradins de la cavea en est une importante, souvent laissée de côté. Ainsi, il apparaît clairement que les théâtres équipés de gradins à marchepied et banquette (Argenton I, Métaponte, Lenzburg, Vidy) sont beaucoup plus "plats» que les constructions "classiques».

La vision forcément réductrice qui pousse à placer chaque monument dans un type à la définition rigide doit être remise en cause.

La diversité constatée entre les édifices prouve les facultés d'adaptation des artisans locaux aux nouvelles techniques apportées par Rome, plutôt que leur incapacité à reproduire un modèle de référence. Elle témoigne de la liberté de choix des matériaux, de la suppression ou du remplacement

IO. Dimensions et localisation de la scène, extension et forme de la cavea, dimensions de l'orchestra, présence ou non d'aditus et de vomitoire en sont quelquesunes. Cf. P. Ciancio Rossetto, G. Pisani Sartorio, Teatri greci e romani, p. 134-145; U. Niffeler, Römisches Lenzburg, p. 165-178; P. Gros, L'architecture romaine, p. 294298; Ch. Landes (éd.), Le goût du théâtre à Rome et en Gaule romaine, p. 25-32.

II. Parmi ceux-ci: Lenzburg (U. Niffeler, Römisches Lenzburg), Argentomagus, SaintMarcel (F. Dumasy, Le théâtre d'Argentomagus) et Avenches (G. Matter, Das römische Theater von Avenches VD/Aventicum, Architektur, Baugeschichte, kulturhistorische Aspekte).

I2. La répartition de ce type de monuments couvre la France, la Suisse, la Belgique, une partie de l'Allemagne et de l'Angleterre. 
de certains éléments constitutifs ${ }^{13}$ et de l'affranchissement des règles déterminant le plan rigoureux du théâtre romain. Cela génère parfois des formes irrégulières, voire inattendues ${ }^{14}$, inexistantes dans le répertoire classique. C'est pourquoi l'aspect et la constitution du monument lausannois ne doivent pas étonner démesurément. Des formes a priori curieuses, de même que la qualité des matériaux mis en œuvre, ne sont plus à considérer uniquement comme des tentatives maladroites de copie des réalisations romaines.

L'évocation du théâtre (romain ou gallo-romain) fait invariablement référence à une construction semi-circulaire garnie de gradins, dévolue à un type de spectacle bien défini (tragédie ou comédie), reportant immanquablement aux origines grecques. De ce fait, les théâtres de province ne sauraient être que des imitations, dont le besoin ne se fait sentir qu'après la conquête. Les indigènes, séduits par les avantages de la vie à la romaine, ne résisteraient pas à l'attrait des monuments publics, particulièrement celui du théâtre et son côté ostentatoire. Si ce genre de raisonnement peut s'appliquer dans certains cas, il faut se garder de le généraliser. Les découvertes de ces dernières années et leurs études plus poussées font une place plus grande aux particularités dont les probables origines traditionnelles locales sont sans doute plus anciennes qu'on ne l'a imaginé jusqu'ici.

Le théâtre est avant tout un concept; il s'agit d'un lieu défini (temporaire ou permanent) où se regroupent des individus pour assister (regarder et écouter) à un évènement public. En ce sens, le théâtre peut s'affranchir d'un catalogue de formes prédéfinies et se limiter - comme le théâtre des origines - à une butte, naturelle ou non; on s'y tient debout ou assis à même le sol, face à un espace scénique de quelques mètres carrés. Ajoutons qu’archéologiquement, il ne faut espérer aucun vestige de tels aménagements.

13. Ainsi, le grand appareil est souvent abandonné au profit de la construction en moellons.

I4. Argentomagus, Antigny, Arleuf, Canouville, Jublains, Trèves, par exemple. 
2.2. Formes et dimensions (fig. 2)

Aucune étude architecturale n'ayant été réalisée pour le théâtre de Lousonna ${ }^{15}$, il n'est pas possible d'en établir un plan général précis. La largeur du monument peut être fixée à 59,50 m sur la base du mur frontal (M8/18/19) dégagé sur sa quasi-totalité. En revanche, on ignore tout de la profondeur de la construction car les murs disparaissent dans l'épaisseur des remblais du talus qu'il n'a pas été possible de fouiller; il n'est pas même certain que ces vestiges aient subsisté.

Tout se complique encore lorsqu'il s'agit de préciser la forme globale de l'édifice. La logique dirige prioritairement vers le demi-cercle prolongé. On aurait alors un monument inscrit à l'intérieur d'un cercle de $59,50 \mathrm{~m}$ dont le centre correspond à celui de l'orchestra ${ }^{16}$. Toutefois, les quelques rangées de gradins conservées ne projettent pas la même image. Distribuées à l'intérieur de secteurs mal définis, elles sont clairement d'orientations divergentes. De plus, elles ne sont comparables ni dans leur construction, ni dans leurs dimensions et, si certains gradins présentent une très légère courbure, la plupart sont parfaitement rectilignes. Dès lors, il est exclu de procéder à une restitution de la répartition des gradins par simple projection symétrique de part et d'autre de l'axe médian.

Ces indications incomplètes et contradictoires poussent à envisager un théâtre de plan polygonal, peut-être irrégulier, plutôt que semi-circulaire, solution de loin la plus courante. Poursuivant cette idée, quelques murs rectilignes, mis au jour lors de travaux anciens ${ }^{17}$, pourraient être intégrés au périmètre du théâtre. Toutefois, le manque de précision concernant ces murs rend conjecturale leur incorporation au monument.

A titre indicatif, sur la base d'une pente constante de la cavea, on peut estimer une hauteur de $5 \mathrm{~m}$ minimum pour le mur périmétral en son point le plus élevé.

15. Plusieurs projets de publication sont en discussion, mais l'intérêt pour les ruines du théâtre est à ce jour insuffisant pour aboutir à une concrétisation.

I6. Ce n'est pas forcément le cas. A Arleuf, monument très intéressant par son plan atypique, une différence a été notée par les fouilleurs entre le centre de l'orchestra et celui de la cavea (L. Olivier, "Le théâtre antique des Bardiaux»).

I7. Travaux d'extraction de sable en 1894. 


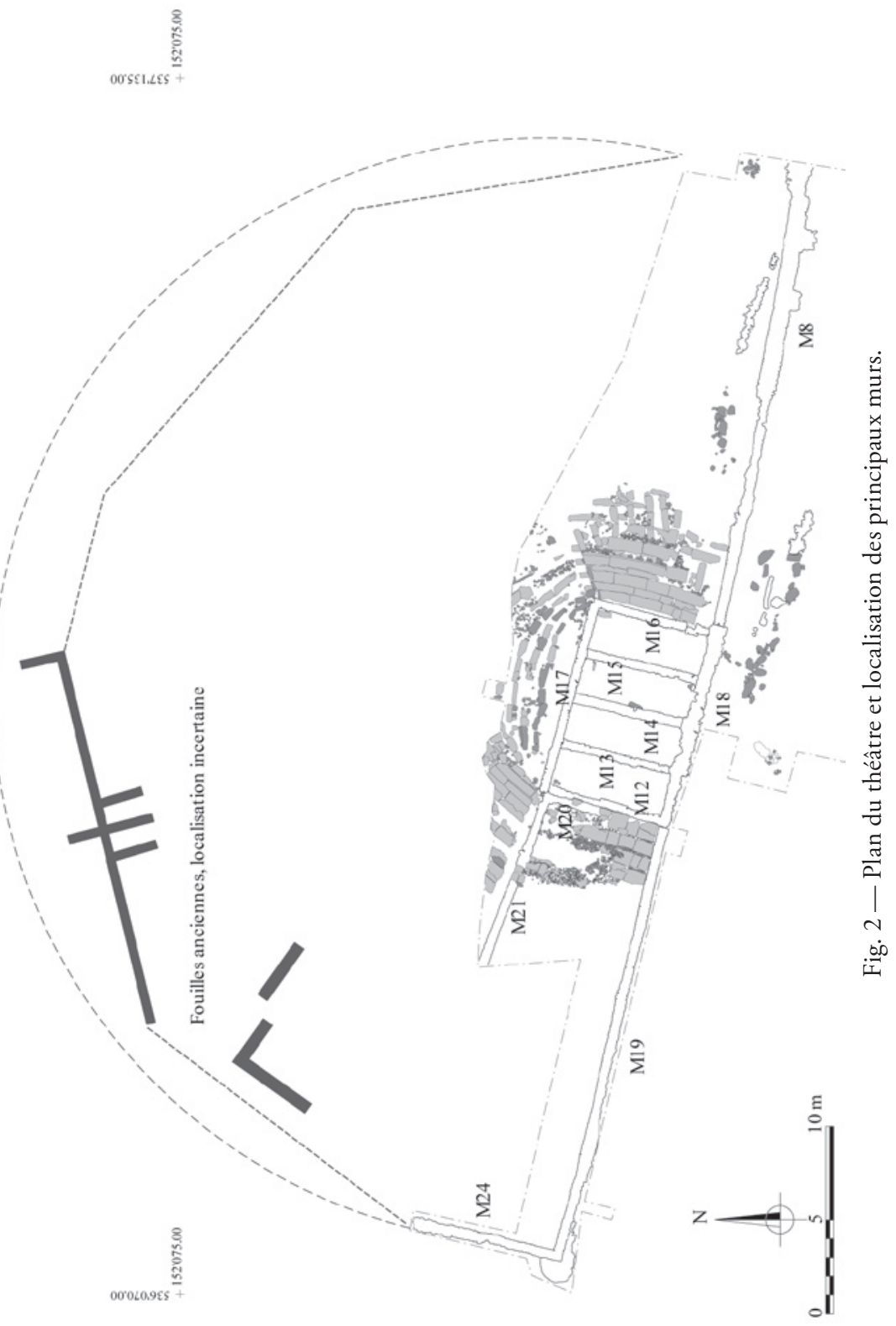




\subsection{Les murs périmétraux}

Le mur de façade n'est ni uniforme, ni homogène. Il est constitué de trois tronçons, respectivement, d'est en ouest: M8, M18 et M19.

Le mur M8 s'élève très légèrement d'ouest en est. Ce mouvement est sensible aussi bien dans l'altitude des fondations que dans celle des arasements. En ne considérant que le niveau de fondation, la pente constatée est de $80 \mathrm{~cm}$ environ sur une distance de $4 \mathrm{~m}$; cette déclivité est nettement inférieure à celle constatée sur les gradins de molasse conservés. Dans sa partie orientale, le mur constitué de galets calcaires se développe sur une épaisseur de $1 \mathrm{~m}$ au niveau des fondations. Il s'agit du tronçon soumis aux plus importantes contraintes, comme l'attestent les restes de deux contreforts sur la face sud, visiblement implantés dès l'origine. Des écarts dans l'épaisseur du mur ainsi qu'un changement de module des matériaux indiquent de possibles réfections antiques. Dans la portion centrale, la construction limitée à une largeur de $50 \mathrm{~cm}$ environ est très irrégulière. Les derniers mètres jusqu'à la jonction avec le mur sud de la scène (M18) sont d'une facture nettement plus soignée. Cette portion présente deux parements propres et un blocage interne maçonné. L'épaisseur est de $60 \mathrm{~cm}$. Les fondations $(40 \mathrm{~cm})$ sont de galets bruts non maçonnés, surmontées de deux assises de pierres taillées sur une face et liées au mortier (env. $30 \mathrm{~cm}$ ) marquant un ressaut. Au sommet, trois assises supplémentaires (env. $30 \mathrm{~cm}$, élévation?) et parementées sur deux faces complètent ces vestiges.

La configuration du mur - élargissement vers la périphérie et pente des fondations - va dans le sens d'une restitution peu élevée, s'abaissant vers l'ouest et peu apte à supporter des contraintes importantes. D'ailleurs, un amoncellement de cailloux et de fragments de mortier, observé en aval du mur, peut être interprété comme les restes d'un effondrement. Un calcul simple effectué lors de la fouille permet de proposer une hauteur minimale d'env. 1,60 m à cet endroit. A son extrémité ouest, la construction se termine par une tête de mur parementée, de finition soignée. Il n'existe aucune trace de chaînage avec M18. Le traitement de cette jonction montre l'antériorité de M8 sur M18.

Le mur M18 (mur sud de soutènement de la scène) est fortement ruiné. Des modifications se perçoivent dans les variations de dimensions. L'extrémité orientale, sans doute l'une des portions les plus récentes, mesure $1 \mathrm{~m}$ d'épaisseur. Effondré, il a été renforcé au cours des dernières 
transformations; cela n'a visiblement pas empêché d'autres éboulements. Ce mauvais état de conservation occulte les relations de ce mur avec les transverses de soutènement de la scène (M12-M16, fig. 2). Quoi qu'il en soit, ce mur, qui avec ses 11,20 m correspond à la largeur de la scène, est totalement indépendant des murs M8 et M19. Cette dernière observation laisse deviner plusieurs aménagements successifs de la scène.

Le mur M19 s'étire en droite ligne sur plus de $24 \mathrm{~m}$. Il est constitué de galets calcaires taillés sur une face, maçonnés et alignés sur deux parements et trois à six assises; le tout repose sur deux assises de galets bruts, non maçonnés. A son extrémité ouest, il est complété par un retour à $90^{\circ}$ en direction du nord (M24). L'angle parfaitement chaîné que forment ces deux murs est consolidé par un puissant contrefort semi-circulaire d'environ $1 \mathrm{~m}$ de rayon. Constitué des mêmes éléments que ceux des murs, cet artifice présente une forte proportion de mortier qui en assure la cohésion. La contemporanéité du contrefort et du mur n'a pas pu être vérifiée. Quoi qu'il en soit, des problèmes de statique sont constatés sur cette construction.

A sa jonction avec le mur de scène M18, on remarque une facture particulièrement soignée - comme pour $\mathrm{M} 8$, à l'est - faite de moellons beaucoup plus gros que ceux utilisés sur le reste de la longueur. Le bas des fondations montre un dénivelé d'est en ouest de 1,10 $\mathrm{m}$. Cette déclivité, comparable à celle observée sur le mur M8, tend à confirmer une configuration topographique en légère cuvette.

Le mur périmétral ouest (M24) a été observé sur une longueur rectiligne de 8,50 m. Contemporain de M19 dont il est entièrement solidaire, il en a exactement les mêmes caractéristiques sur les quatre premiers mètres. Ensuite, l'utilisation de pierres plus grosses s'accompagne d'une brusque remontée du niveau d'implantation. On ignore si son orientation vers le nord se poursuit au-delà du tronçon dégagé. Son équivalent symétrique à l'est, sans doute situé hors périmètre de fouille, n’a pas été observé.

De manière générale, les murs périmétraux ne semblent pas avoir fait l'objet d'un traitement de surface particulier. 


\subsection{La cavea}

a) Les remblais

Installé à l'arrière contre les flancs de la colline et dans une légère cuvette, le théâtre s'avance au sud sur un remblai artificiel, maintenu par les murs frontaux sur plus de $59 \mathrm{~m}$. Les matériaux constitutifs de cette terrasse proviennent sans doute de l'excavation effectuée dans le flanc de la colline, pour les niveaux supérieurs. Les niveaux inférieurs appartiennent probablement aux constructions de terre et bois ayant précédé la construction du théâtre. Cette proposition est confortée par l'étude du mobilier ${ }^{18}$. Il est toutefois impossible d'être plus précis, la vision en coupe transversale (nord-sud) étant largement incomplète. Le remblai est indispensable à une accentuation de la pente et au soutènement des rangées de gradins rayonnants de part et d'autre de la scène, en particulier aux deux extrémités frontales de la cavea.

\section{b) Les gradins}

L’ensemble des gradins conservés a été divisé arbitrairement en trois zones distinctes présentant une certaine uniformité interne. Ces surfaces sont indépendantes les unes des autres et vraisemblablement trapézoïdales, sans que l'on puisse pour autant parler de cunei. L'existence même d'une telle division, issue du plan classique, n'est pas certaine sur le théâtre de Vidy.

L'orientation, la direction, le mode d'assemblage et la dimension des blocs constituant les gradins varient selon les zones considérées. Ce constat pousse à imaginer un monument asymétrique ou ayant subi des transformations au cours de son utilisation, expliquant son aspect actuel hétéroclite.

Les gradins sont rectilignes à l'ouest et au centre ce qui suppose une cavea polygonale. Cependant, à l'est, les rangées sont très légèrement curvilignes selon un rayon beaucoup trop important pour pouvoir être respecté sur l'ensemble de la cavea; ceci implique des ruptures dans l'orientation des gradins.

18. Cf. infra. 
Autre différence notable révélée par les fouilles, les gradins de l'est reposent sur des substructures de blocs molassiques. Un premier assemblage est disposé de manière concentrique; lui est superposée une seconde combinaison, cette fois-ci rayonnante, sur laquelle s'appuient les dalles des gradins. Les observations manquent pour préciser si ces différents systèmes sont contemporains, correspondent à un changement du type de gradins ou encore sont l'image de réfections opérées à la suite de tassements de l'ensemble. Cet agencement n'a pas été observé ailleurs. La pente des blocs, mal conservés, n'a pu être mesurée et aucune trace d'encoche n'a été relevée sur les substructures. En outre, les gradins de l'est se situent $40 \mathrm{~cm}$ au-dessous de leurs correspondants au centre et à l'ouest. Ces diverses observations sont vraisemblablement le reflet de l'existence de plusieurs phases de construction. Les gradins rectilignes viendraient remplacer les gradins semi-circulaires avec toutes les conséquences que cela implique sur la forme générale du monument.

A l'ouest, les murs M20 et M21, chaînés et de facture identique, forment entre eux une structure en $\mathrm{L}$ difficilement assimilable au reste du monument dans son état final (fig. 2). Il n'est pas possible de déterminer si M20 sert de séparation entre la cavea et l'orchestra à un moment où cette dernière n'est pas entièrement occupée par la scène. Comme le laisse supposer la configuration actuelle, ces murs sont peut-être à mettre en relation avec une phase antérieure du théâtre qu'on ne peut préciser pour l'instant.

Bien que de réalisation différente selon les secteurs considérés, tous les gradins conservés sont constitués de deux blocs de molasse ${ }^{19}$ : un marchepied et une banquette. Celle-ci vient s'insérer dans une feuillure à l'arrière de la dalle marchepied. Cet assemblage, qui assure une bonne stabilité à l'ensemble, s'oppose à la technique «monobloc» largement plus répandue. Il permet par ailleurs de considérables économies de pierre ${ }^{20}$. Le système adopté ici, en ménageant des espaces de circulation entre chaque rang de $50 \mathrm{~cm}$ de largeur, fournit aux spectateurs un

19. C'est le cas également à Lenzburg (U. Niffeler, Römisches Lenzburg, p. 107).

20. Un calcul comparatif simplifié avec le théâtre de Vienne montre que le volume de pierre nécessaire pour une même surface utilisable de gradins peut varier considérablement; dans notre exemple, jusqu'à $25 \%$ supérieur! Les chiffres utilisés (dimensions des blocs des gradins) pour ce calcul sont tirés de J. Formigé, Le théâtre romain de Vienne et des documents de fouilles du théâtre de Vidy. Ils ne tiennent pas compte de la pente de la cavea ni de la hauteur des murs. 
confort qu'ils ne trouvaient pas à Argenton où une disposition comparable est avérée ${ }^{21}$. En outre, cela a peut-être permis de s'affranchir de l'installation d'une précinction et prouve que le théâtre de Vidy n'est pas une construction misérable.

La réalisation d'une cavea avec ce type de gradins va de pair avec une pente faible. C'est le cas à Vidy ainsi qu'à Lenzburg ${ }^{22}$. La différence de hauteur constatée entre deux rangs est d'env. $15 \mathrm{~cm}$, soit une demi-tête environ. Si cette configuration ne favorise guère la qualité du champ de vision, elle est par contre source d'économie de temps et de travail lors de la construction.

Au-delà des gradins conservés, les fouilles n'ont livré aucune indication quant à l'organisation de la cavea dans la partie haute du monument. Plusieurs hypothèses sont envisageables. Soit les gradins de molasse ont été entièrement récupérés, soit les gradins étaient de bois et ont disparu. Une troisième solution serait l'absence de gradins, remplacés par de simples talus comme dans les tout premiers théâtres. Aucun élément ne permet de trancher aujourd'hui.

\subsection{L'orchestra}

L'orchestra correspond à un demi-cercle prolongé de $11 \mathrm{~m}$ de diamètre et de 10,90 m de profondeur. Cette surface est presque entièrement occupée par la scène qui s'étire latéralement jusqu'aux premiers gradins. Seule la partie semi-circulaire (nord) est dégagée. On y trouve une multitude de blocs d'origines diverses, alignés sur trois arcs de cercle. Le sommet de ces blocs correspond probablement au niveau de circulation de l'orchestra. Certains blocs portent des inscriptions; souvent de qualité médiocre, elles ont presque totalement disparu. Ces inscriptions sont à considérer comme des marquages au sol, signifiant des espaces réservés à l'installation de sièges pour quelques notables du vicus ${ }^{23}$.

2I. Pour le théâtre I, cf. F. Dumasy, Le théâtre d'Argentomagus (Saint-Marcel, Indre), p. 191.

22. C'est le cas également à Argenton, sur le premier théâtre, F. Dumasy, Le théâtre d'Argentomagus (Saint-Marcel, Indre), p. 235. A Lenzburg, au niveau des fondations des murs du vomitorium 2, elle est de $10^{\circ}-12^{\circ}$. Cf. U. Niffeler, Römisches Lenzburg. A Vidy, elle est de $8^{\circ}-10^{\circ}$.

23. Une inscription de ce type est connue à Lopodunum, Bade-Wurtemberg (B. Rabold, C. S. Sommer, Lopodunum 98, vom Kastell zur Stadt, p. 15). 
La plus "lisible» des inscriptions fait référence à la corporation des charpentiers (tignarii) dont elle souligne l'importance ${ }^{24}$. Plusieurs hypothèses peuvent expliquer la présence de cette inscription, la plus probable étant que l'on ait accordé des places de choix à certains membres des corporations ayant participé à la construction du théâtre. En effet, le charpentier gallo-romain (tignarius ou faber), très polyvalent, savait également travailler la pierre. La corporation des charpentiers a peut-être aussi soutenu financièrement la construction du théâtre.

Cette découverte apporte un nouvel éclairage sur le travail des artisans en confirmant l'existence de plusieurs corporations œuvrant dans une ville de petite taille certes, mais très active.

\subsection{La scène}

On cherchera vainement parmi les théâtres gallo-romains de la Gaule du Nord ou de Belgique le vaste bâtiment de scène et sa scaenae frons caractéristique des édifices romains. L'économie d'une telle construction ne fait pas exception à Vidy.

Les restes de la scène se présentent sous la forme d'un quadrilatère de $7 \mathrm{~m}$ x $11 \mathrm{~m}$ oblitérant l'orchestra à plus de $75 \%$. Elle est délimitée par quatre murs de pierre maçonnés, peut-être surmontés d'une estrade de bois. Cette construction comporte en outre trois murs internes perpendiculaires à la grande longueur.

La scène dans son état visible est le fruit d'une construction d'un seul jet. Elle constitue une unité homogène et indépendante, que l'on peut considérer comme un ajout postérieur au premier état. Cette impression est confirmée par l'analyse du mobilier ${ }^{25}$ et la finition particulièrement soignée des têtes des murs frontaux jouxtant la scène de part et d'autre. Ces derniers étaient en place avant l'installation de la scène actuelle.

Dans sa version définitive, elle ne devait guère s'élever de 50 à $60 \mathrm{~cm}$ au-dessus du sol afin de ne pas gêner la vision des spectateurs des premiers rangs.

24. La seule décryptée à ce jour par Regula Frei-Stolba, Institut d'Archéologie et des Sciences de l'Antiquité, Université de Lausanne (cf. F. Eschbach, «Les charpentiers de Lousonna-Vidy» et Lausanne-Vidy, les fouilles du théâtre.

25. Les murs de la scène sont implantés dans des couches ayant livré du mobilier du III siècle apr. J.-C. 
Impossible latéralement, l'accès à la scène se faisait obligatoirement par le sud, peut-être au moyen d'un escabeau de bois. Ce passage, l'absence de lien entre les différents segments du mur frontal et celui de la scène ainsi que leur construction relativement légère poussent à imaginer un «mur de scène» peu élevé ou inexistant.

\subsection{Accès extérieurs et circulation interne}

L'arrivée du flot de spectateurs vers le monument se faisait par un cheminement dont aucune trace n'a été perçue dans l'espace situé à moins de $50 \mathrm{~m}$ au sud du mur frontal. Le théâtre de Vidy était sans doute situé non loin des principales voies en provenance de l'est vers le vicus.

L'approche du monument s'organisait perpendiculairement depuis l'une de ces voies, soit par le nord, soit par le sud. L'organisation interne du théâtre et sa situation à flanc de coteau ne laissent que peu de choix pour la localisation des accès. A moins d'imaginer un peu vraisemblable passage par la scène, l'entrée ne peut se faire que sur les côtés ou par l'arrière; les fouilles n'ayant pas atteint ces zones, l'incertitude quant à la forme des accès (escaliers, rampes ou accès à niveau) demeure.

La solution aux problèmes de la circulation interne du public ne peut être évoquée de manière plus claire. L'existence de passages se limite aux endroits où l'on note une interruption des gradins de molasse. La localisation la plus vraisemblable, archéologiquement parlant, se situe sur le côté est, contre le mur frontal (M8). A cet endroit, on constate un vide d'environ $70 \mathrm{~cm}$ de largeur entre l'extrémité des gradins et le mur frontal. D'autres passages rayonnants sont envisageables au-dessus du mur M21 ainsi que dans l'angle nord-est de la scène, mais les preuves manquent.

\section{Chronologie, datation}

Stratigraphiquement, le théâtre se superpose à une zone d'habitat, quartiers périphériques du vicus de Lousonna édifiés au cours du $\mathrm{I}^{\mathrm{er}}$ siècle apr. J.-C. ${ }^{26}$ Une partie des constructions est probablement rasée pour faire place - au début du $\mathrm{II}^{\mathrm{e}}$ siècle apr. J.-C. - au nouvel édifice, qui en

26. F. Eschbach, C. Brunetti, Vidy, av. des Figuiers 41 et 43. 
respecte les alignements. L'abandon du théâtre, qui reste difficile à situer, n'est pas suivi par de nouvelles occupations.

Comme on l'a vu, les fouilles du monument ne concernent que des surfaces réduites. Les rares couches fouillées sont pauvres en mobilier datant, exception faite des monnaies dont le nombre dépasse la cinquantaine pour les seules zones explorées. L'étude de la céramique ${ }^{27}$ et celle des monnaies ${ }^{28}$ situent la construction du théâtre au début du $\mathrm{II}^{\mathrm{e}}$ siècle apr. J.-C., période de prospérité pour le vicus de Lousonna. Elles permettent également de proposer un réaménagement partiel du monument dans la première moitié du III $\mathrm{e}^{\mathrm{e}}$ siècle, ainsi qu'une rénovation tardive au cours du même siècle.

Quelques indices ponctuels repérés ici et là (cf. supra) prouvent un fonctionnement du théâtre en plusieurs phases. Ces étapes ne sont toutefois pas datables avec suffisamment de précision et sont trop fragmentaires pour permettre une mise en phases élaborée; les divers évènements présentés dans le tableau (ci-dessous) ne sont pas forcément contemporains et il est probable que plusieurs phases nous échappent.

\begin{tabular}{|l|l|l|l|l|l|}
\hline Phase & Scène & Gradins ouest & orchestra & Gradins est & Datation \\
\hline I & $\begin{array}{l}\text { petite scène } \\
\text { (bois?) }\end{array}$ & $\begin{array}{l}\text { niveau } \\
\text { profond } \\
\text { M20 et M21 } \\
\text { délimitent le } \\
\text { secteur }\end{array}$ & libre & $\begin{array}{l}\text { premiers } \\
\text { gradins et } \\
\text { substruc- } \\
\text { tures de } \\
\text { molasse }\end{array}$ & $\begin{array}{l}\text { début } \\
\mathrm{II}^{\mathrm{e}} \text { s. }\end{array}$ \\
\hline II & ?? & $\begin{array}{l}\text { abandon } \\
\text { de M20 et } \\
\text { M21, gradins } \\
\text { recouvrant } \\
\text { les niveaux } \\
\text { inférieurs }\end{array}$ & $\begin{array}{l}\text { apport de } \\
\text { remblai, } \\
\text { surélévation }\end{array}$ & ?? & $\begin{array}{l}\text { première } \\
\text { moitié } \\
\text { III s. }\end{array}$ \\
\hline III & $\begin{array}{l}\text { agrandis- } \\
\text { ?ement } \\
\text { maximal }\end{array}$ & $\begin{array}{l}\text { ?? } \\
\text { fortement } \\
\text { empiétée } \\
\text { par la scène }\end{array}$ & $\begin{array}{l}\text { abandon des } \\
\text { premières } \\
\text { rangées }\end{array}$ & $\begin{array}{l}\text { seconde } \\
\text { moitié } \\
\text { III } s .\end{array}$ \\
\hline
\end{tabular}

27. Réalisée par Caroline Brunetti, Archéodunum S.A.

28. Réalisée par Anne Geiser et Yves Mühlemann, Cabinet des médailles, Musée Cantonal d'Archéologie et d'Histoire, Lausanne. 


\section{Synthèse et perspectives}

Le théâtre gallo-romain de Lousonna est édifié vraisemblablement au début du $\mathrm{II}^{\mathrm{e}}$ siècle apr. J.-C., en périphérie du vicus dans un quartier d'habitat dont les alignements urbanistiques seront respectés. Les directeurs du projet ont su mettre à profit une configuration topographique favorable, permettant de notables économies lors de la mise en œuvre. Les techniques de construction employées et les dimensions en font un monument modeste comparativement aux trois autres édifices connus à ce jour en Suisse. Bien que fortement ruiné, le théâtre de Vidy laisse encore voir nombre de particularités qui rendent cet édifice extrêmement difficile à intégrer dans une typologie, par ailleurs en perpétuelle évolution.

Les interventions archéologiques superficielles ont permis d'entrevoir plusieurs phases de fonctionnement. En revanche, il n'a pas été possible de déterminer la forme générale du monument que seules des fouilles plus étendues et, pour tout dire, fort improbables, permettraient de découvrir.

Nombre d'aspects de l'étude de ce théâtre n'ont pu être abordés dans le cadre du présent article. Je pense ici notamment à l'étude architecturale, liée à une analyse des maçonneries qui autoriserait l'ébauche de quelques propositions de restitution ${ }^{29}$. Il serait alors possible de calculer le nombre de places disponibles dans la cavea et ainsi d'avoir une idée du rayonnement des manifestations antiques. Préciser de quel type étaient celles-ci reste également une question à résoudre. Il conviendrait, en outre, de replacer le monument dans le contexte historique du vicus. Une étude métrologique approfondie et une analyse épigraphique plus poussée seraient aussi à envisager. La comparaison avec d'autres monuments pourrait alors être effectuée avec plus de rigueur.

Hormis le caractère exceptionnel de la découverte - ce n'est pas tous les jours qu'on a la chance de dégager un théâtre - ce monument possède des spécificités qui en renforcent l'intérêt.

29. Un intéressant travail a cependant été réalisé sur ce sujet; il a abouti à la fabrication d'une maquette du théâtre, aujourd'hui présentée au Musée romain de LausanneVidy. Cf. B. Julita, L'évolution architecturale des théatres et le théatre gallo-romain de Lousonna-Vidy. 
Par ses dimensions réduites, sa forme générale déroutante et par sa conception même, le théâtre de Vidy se distingue notablement des autres constructions du même type connues en Suisse et hors des frontières. Une telle forme est-elle l'expression de la ruralité, d'une «romanisation" incomplète, d'une inaptitude technologique à reproduire un modèle romain plus abouti ? Prouve-t-elle le côté archaïque de la construction ou, au contraire, traduit-elle une adaptation plus nuancée que celle habituellement admise de la conception traditionnelle des lieux de réunions à celle récemment importée? ${ }^{30}$ Les réalisations architecturales de l'agglomération au II siècle apr. J.-C., publiques et privées, prouvent que les capacités des architectes et des artisans ne sont pas en cause.

Ces caractéristiques sont une chance dans la mesure où elles invitent à reprendre des dossiers aussi fondamentaux que ceux des origines et de la détermination du théâtre en Gaule. Par suite, il devient indispensable de redéfinir les liens très complexes existants entre la forme, la fonction et l'interprétation d'un tel monument; sont donc concernés au premier titre les problèmes d'architecture et du type de "spectacle" proposé dans les théâtres gallo-romains. C'est également l'opportunité de revoir une série de questions élémentaires sous un éclairage nouveau (choix des matériaux et du site, urbanisme, etc.). Ne serait-ce que du point de vue du monument lui-même, cette découverte mérite qu’on lui réserve toute notre attention.

En outre, la mise au jour des vestiges, en décembre 1998, constitue un apport majeur pour la compréhension de l'histoire du vicus. En effet, la signification de la présence même d'un tel monument et sa localisation à l'est du Flon remettent en cause un certain nombre d'idées reçues. Il s'agit, entre autres, de l'extension insoupçonnée du vicus dans cette direction et de l'existence d'une trame urbaine orthogonale précoce, dès le début du Irer siècle apr. J.-C.

De plus, les inscriptions révélées par la fouille sont du plus grand intérêt. Ces marquages au sol gravés sur des blocs de pierre disposés dans la partie du monument réservée aux notables permettent non seulement d'aborder l'aspect de l'économie du théâtre mais également celui de l'organisation sociale du vicus.

30. A l'image de ce qui se passe pour la religion gallo-romaine, considérée comme une transposition libre de celle des Romains, conjointement à la survivance de croyances gauloises. 
Le côté inopiné de la découverte n'est pas anodin; il impose un nouveau regard sur le discours qui peut être tenu en «l'absence» de découverte.

Enfin, la façon dont sont actuellement conservés les murs dégagés en 2000 - sous le bâtiment ayant pris place sur les ruines - soulève le problème épineux de la conservation et du devenir du théâtre, suscitant un débat autour des solutions proposées. Les vestiges existants, s'ils sont bien à l'abri des ravages météorologiques, se situent également à l'écart des visiteurs potentiels ${ }^{31}$. Peu présentables pour l'heure, ces vénérables ruines mériteraient d'être mises en valeur et rendues accessibles sous certaines conditions.

On le voit, cette découverte suscite de nombreux débats et la reprise de certains dossiers que l'on croyait bouclés. Les problèmes posés ici et ceux que poseront les futures découvertes montrent qu'il existe encore un vaste champ d'investigation dans le domaine de l'étude des édifices de spectacle et c'est tant mieux!

François Eschbach Archéodunum S.A.

3I. Un public nombreux et intéressé comme l'a prouvé le succès des journées portes ouvertes organisées lors des fouilles. 


\section{BIBLIOGRAPHIE}

\section{Sources}

Vitruve, De Architectura, III, Paris, Les Belles Lettres, 2003.

\section{Etudes}

Ciancio Rossetto, Paola, Pisani Sartorio, Giuseppina, Teatri greci e romani. Alle origini del linguaggio rappresentativo, Torino, Censimento analitico, 1994.

Dumasy, Françoise, Le théâtre d'Argentomagus (Saint-Marcel, Indre), Paris, Maison des Sciences de l'Homme, 2000.

Eschbach, François, "Les charpentiers de Lousonna-Vidy", Mémoire Vive, 9 (2001), p. 43-44.

—, "Découverte d'un nouveau quartier à Lousonna-Vidy", Mémoire Vive, 10 (2002), p. 37-39.

—, Lausanne-Vidy, les fouilles du théâtre, Rapport de fouilles dactylographié, Gollion, 2005.

Eschbach, François, Brunetti, Caroline, Vidy, av. des Figuiers 41 et 43, Rapport de fouilles dactylographié, Gollion, 2007.

Formigé, Jules, Le théâtre romain de Vienne, Vienne, Audin, 1949.

Gros, Pierre, L'architecture romaine 1: Les monuments publics, Paris, Picard, 1996.

Julita, Bastien, L'évolution architecturale des théatres et le théatre galloromain de Lousonna-Vidy: essai de restitution, mémoire de licence soutenu à la Faculté des lettres de l'Université de Lausanne, 2005 (non publié).

Landes, Christian (éd.), Le goût du théâtre à Rome et en Gaule romaine, Lattes, Editions Imago, 1989. 
Matter, Georg, Das römische Theater von Avenches VD/Aventicum, Architektur, Baugeschichte, kulturhistorische Aspekte, Lausanne, Cahiers d'archéologie romande, 2009 (Cahiers d'archéologie romande 114, Aventicum XV).

Niffeler, Urs, Römisches Lenzburg: Vicus und Theater, Brugg, Gesellschaft Pro Vindonissa, 1988.

Olivier, Lucien, "Le théâtre antique des Bardiaux", in Spectacula II. Le théâtre antique et ses spectacles. Actes du colloque tenu au musée archéologique Henri-Prades de Lattes les 27, 28, 29 et 30 avril 1989, éd. Christian Landes, Lattes, 1992, p. 57-62.

Rabold, Britta, Sommer, C. Sebastian, Lopodunum 98, vom Kastell zur Stadt, Stuttgart, 1998.

Crédits iconographiques

Fig. 1:

DAO: E. Soutter, Archéodunum S.A.

Fig. 2:

DAO: F. Eschbach, Archéodunum S.A. 